PROCEEDINGS OF THE

AMERICAN MATHEMATICAL SOCIETY

Volume 127, Number 5, Pages 1269-1274

S 0002-9939(99)04775-9

Article electronically published on January 27, 1999

\title{
ON SWAN CONDUCTORS FOR BRAUER GROUPS OF CURVES OVER LOCAL FIELDS
}

\author{
TAKAO YAMAZAKI
}

(Communicated by David E. Rohrlich)

\begin{abstract}
For an element $w$ of the Brauer group of a curve over a local field, we define the "Swan conductor" $\operatorname{sw}(w)$ of $w$, which measures the wildness of the ramification of $w$. We give a relation between $\operatorname{sw}(w)$ and Swan conductors for Brauer groups of henselian discrete valuation fields defined by Kato.
\end{abstract}

\section{INTRODUCTION}

Let $k$ be a complete discrete valuation field with finite residue field $F$ of characteristic $p$. Let $O_{k}$ be the ring of integers in $k$. Let $X$ be a projective smooth geometrically connected curve over $k$. There is a canonical pairing (cf. [4], [5], Section 9)

$$
\langle,\rangle_{X}: \operatorname{Pic}(X) \times \operatorname{Br}(X) \rightarrow \mathbf{Q} / \mathbf{Z},
$$

described as follows: For a closed point $x \in X$ and an element $w \in \operatorname{Br}(X)$, we have the localization $w_{x} \in \operatorname{Br}(\kappa(x))$ of $w$ at $x$. Since $\kappa(x)$ is a finite extension of $k$, we have $\operatorname{Cor}_{\kappa(x) / k}\left(w_{x}\right) \in \operatorname{Br}(k) \cong \mathbf{Q} / \mathbf{Z}$, where the last isomorphism is given by local class field theory. Then we define the pairing (1) by

$$
\left\langle\sum_{x} n_{x}[x], w\right\rangle_{X}=\sum_{x} n_{x} \operatorname{Cor}_{\kappa(x) / k}\left(w_{x}\right) \in \mathbf{Q} / \mathbf{Z},
$$

where $[x]$ denotes the class of $x$ in $\operatorname{Pic}(X)$ and $n_{x} \in \mathbf{Z}$.

The pairing (1) induces an isomorphism

$$
\operatorname{Br}(X) \stackrel{\cong}{\rightrightarrows} \operatorname{Hom}_{c}(\operatorname{Pic}(X), \mathbf{Q} / \mathbf{Z}) .
$$

Here $\mathrm{Hom}_{c}$ denotes the group of all continuous homomorphisms of finite order, and $\operatorname{Pic}(X)$ is endowed with a certain topology defined in [5], 9.4 (cf. Lemma 3.2). This result was first proven by Lichtenbaum, ignoring the $p$-primary part when $\operatorname{char}(k)=p>0$, and more recently the general case was proven by Saito (cf. loc. cit.).

Taking a model of $X$ over $O_{k}$, we can define a decreasing filtration $U^{m} \operatorname{Pic}(X) \subset$ $\operatorname{Pic}(X)(m>0)$. For $w \in \operatorname{Br}(X)$, we define the Swan conductor $\operatorname{sw}(w)$ of $w$ to be the minimal number $m$ such that the map $\langle\cdot, w\rangle_{X}$ annihilates $U^{m+1} \operatorname{Pic}(X)$ (cf. Definition 3.1). This Swan conductor measures the ramification of $w$.

Received by the editors May 5, 1997 and, in revised form, August 8, 1997.

1991 Mathematics Subject Classification. Primary 11G20, 11S15.

(C)1999 American Mathematical Society 
On the other hand, for an element $w$ of the Brauer group of a henselian discrete valuation field $\Lambda$, Kato defined the Swan conductor $\operatorname{sw}_{\Lambda}(w)$ of $w$, which again measures the ramification of $w$ (cf. Definition 2.1). A certain description of those Swan conductors in terms of division algebras is given in [6] (cf. Theorem 2.2).

The following is the main theorem of this note:

Theorem 1.1. Let $\mathfrak{X}$ be a regular model of $X$ over $O_{k}$ (cf. the beginning of Section 3). For each generic point $\eta \in Y=\left(\mathfrak{X} \otimes_{O_{k}} F\right)_{\text {red }}$, let $K_{\eta}$ be the fraction field of the henselization of $O_{\mathfrak{X}, \eta}$ and $e_{\eta}$ the multiplicity of $\{\eta\}$ in the divisor $\mathfrak{X} \otimes F$. Then, for $w \in \operatorname{Br}(X)$ we have

$$
\operatorname{sw}(w)=\sup \left\{\left[\operatorname{sw}_{K_{\eta}}\left(w_{K_{\eta}}\right) / e_{\eta}\right] \mid \eta \text { runs over the generic points of } Y\right\},
$$

where $w_{K_{\eta}}$ is the natural image of $w$ in $\operatorname{Br}\left(K_{\eta}\right)$, and [ ] denotes the least integer function.

The author would like to express gratitude to K. Kato and S. Saito for their valuable advice. He also thanks K. Sato for his comments and K. Bannai who carefully read the manuscript. He is grateful to the referee, whose comments improved this paper very much. He is supported by JSPS Research Fellowship for Young Scientists.

\section{Review on Swan conductors of Kato}

In this section, we briefly recall Swan conductors for Brauer groups of henselian discrete valuation fields, which were defined by Kato (cf. [3], Proposition 6.5). Let $\Lambda$ be a henselian discrete valuation field with residue field $E$ such that $\operatorname{char}(E)=$ $p>0,\left[E: E^{p}\right]=p$, and $p \operatorname{Br}(E) \neq 0$. Cup products

$$
H^{1}(\Lambda, \mathbf{Z} / m \mathbf{Z}(1)) \times H^{2}(\Lambda, \mathbf{Z} / m \mathbf{Z}(1)) \rightarrow H^{3}(\Lambda, \mathbf{Z} / m \mathbf{Z}(2)) \quad(m>0)
$$

induce a pairing

$$
\langle,\rangle_{\Lambda}: \Lambda^{*} \times \operatorname{Br}(\Lambda) \rightarrow H^{3}(\Lambda, \mathbf{Q} / \mathbf{Z}(2)) .
$$

Here, if $\operatorname{char}(\Lambda)=0, \mathbf{Z} / m \mathbf{Z}(q)$ is defined to be the usual Tate twist of the constant sheaf $\mathbf{Z} / m \mathbf{Z}$. If $\operatorname{char}(\Lambda)=p$, write $m=p^{s} m^{\prime}$ with $s \geq 0, p \not \backslash m^{\prime}$. Then $\mathbf{Z} / m \mathbf{Z}(q)$ denotes the object $\mathbf{Z} / m^{\prime} \mathbf{Z}(q) \oplus W_{s} \Omega_{\Lambda, \log }^{q}[-q]$ of the derived category of abelian sheaves on $\operatorname{Spec}(\Lambda)_{e t}$, where $\mathbf{Z} / m^{\prime} \mathbf{Z}(q)$ is defined to be the usual Tate twist and $W_{s} \Omega_{\Lambda, l o g}^{q}$ is the logarithmic part of the de Rham-Witt complex $W_{s} \Omega_{\Lambda}^{q}$. The group $H^{3}(\Lambda, \mathbf{Q} / \mathbf{Z}(2))$ is defined as the inductive limit of $H^{3}(\Lambda, \mathbf{Z} / m \mathbf{Z}(2))$.

Definition 2.1. For $w \in \operatorname{Br}(\Lambda)$, we define the Swan conductor $\operatorname{sw}_{\Lambda}(w)$ of $w$ to be the non-negative integer

$$
\inf \left\{m \mid \operatorname{ker}\left(\langle\cdot, w\rangle_{\Lambda}\right) \supset U_{\Lambda}^{(m+1)}\right\},
$$

where $U_{\Lambda}^{(m)}$ is the $m$-th unit group of $\Lambda$.

A description of those Swan conductors in terms of division algebras is given by the following theorem, which was proven in [6].

Theorem 2.2. Let $\Lambda$ be as above and $w \in \operatorname{Br}(\Lambda)$. Let $\hat{\Lambda}$ be the completion of the strict henselization of $\Lambda$, and $D$ the division algebra over $\hat{\Lambda}$ corresponding to the natural image $w_{\hat{\Lambda}}$ of $w$ in $\operatorname{Br}(\hat{\Lambda})$. 
(i) The order of $w_{\hat{\Lambda}}$ is equal to $[D: \hat{\Lambda}]^{1 / 2}$ which is equal to $p^{n}$ for some nonnegative integer $n$.

(ii) For any subset $S$ of $D^{*}$, we write

$$
t_{D}(S)=\inf \left\{\operatorname{ord}_{D}\left(a b a^{-1} b^{-1}-1\right) \mid a, b \in S\right\},
$$

where $\operatorname{ord}_{D}$ denotes the normalized valuation on $D$. For $j=0,1, \ldots, n-1$, put

$$
\begin{gathered}
t_{j}=\sup \left\{t_{D}\left(D^{\prime *}\right) \mid D^{\prime} \text { satisfies conditions below }\right\} \\
D^{\prime} \quad \text { is a division algebra, } \\
\hat{\Lambda} \subset D^{\prime} \subset D \\
{\left[D^{\prime}: \text { center of } D^{\prime}\right]=p^{2 j+2}} \\
{\left[\text { center of } D^{\prime}: \hat{\Lambda}\right]=p^{n-j-1} .}
\end{gathered}
$$

Then we have

$$
\mathrm{sw}_{\Lambda}(w)=\frac{t_{0}}{p^{n-1}}+\sum_{j=1}^{n-1} \frac{(p-1) t_{j}}{p^{n-j}} .
$$

Proof. Since the residue field of $\hat{\Lambda}$ is separably closed, (i) is deduced from [6], Proposition 2.1. (Note that the condition $(*)$ in $[6]$ is automatically satisfied.) By [3], Lemma 6.2, we have $\operatorname{sw}_{\Lambda}(w)=\operatorname{sw}_{\hat{\Lambda}}\left(w_{\hat{\Lambda}}\right)$. (Though the Brauer group of the residue field of $\hat{\Lambda}$ is trivial, the definition of $\operatorname{sw}_{\hat{\Lambda}}\left(w_{\hat{\Lambda}}\right)$ is given in [3].) Hence (ii) is deduced from [6], Theorem 5.1.

\section{Proof of Theorem 1.1}

Let $p, k, O_{k}, F$ and $X$ be as in Section 1. Due to [1] and [2], there exists a scheme $\mathfrak{X}$ over $O_{k}$ which satisfies the following property: $\mathfrak{X}$ is a two-dimensional regular proper flat shceme over $O_{k}, \mathfrak{X} \otimes O_{k} k \cong X$ and $Y=\left(\mathfrak{X} \otimes O_{k} F\right)_{\text {red }}$ is a geometrically connected proper one-dimensional scheme over $F$ whose irreducible components are all regular and which has ordinary double points as singularities at worst. Let $j: X \rightarrow \mathfrak{X}$ and $i: Y \rightarrow \mathfrak{X}$ be the inclusion morphisms.

We use the following conventions.

Let $Y_{0}$ (resp. $Y_{1}$ ) be the set of all closed (resp. generic) points of $Y$.

As in Theorem 1.1, for $\eta \in Y_{1}$, let $K_{\eta}$ be the fraction field of the henselization of $O_{\mathfrak{X}, \eta}$ and $e_{\eta}$ the multiplicity of $\{\bar{\eta}\}$ in the divisor $\mathfrak{X} \otimes F$. Similarly, for a closed point $x \in X_{0}$, let $K_{x}$ be the fraction field of the henselization of $O_{X, x}$.

Let $y \in Y_{0}$. Let $A_{y}$ be the henselization of $O_{\mathfrak{X}, y}, R_{y}=A_{y} \otimes_{O_{k}} k$, and $K_{y}$ be the fraction field of $A_{y}$. Let $Y_{1}^{y}$ denote the set of all height one prime ideals in $A_{y}$ lying over some element of $Y_{1}$. (Note that the cardinality of $Y_{1}^{y}$ is 1 or 2.) Similarly, let $X_{0}^{y}$ denote the set of all closed points in $X$ whose closure in $\mathfrak{X}$ includes $y$.

For $\eta \in Y_{1}$ and $y \in\{\bar{\eta}\} \cap Y_{0}$, there is a unique element $\eta_{y}$ of $Y_{1}^{y}$ lying over $\eta$. Let $O_{K_{\eta_{y}}}$ be the henselization of $A_{y}$ at $\eta_{y}$, and $K_{\eta_{y}}$ the fraction field of $O_{K_{\eta_{y}}}$.

Now we define a decreasing filtration on $\operatorname{Pic}(\mathfrak{X})$ and $\operatorname{Pic}(X)$. Fix a prime element $\pi$ of $k$. For $m>0$, let $\mathfrak{X}_{m}=\mathfrak{X} \otimes_{O_{k}}\left(O_{k} / \pi^{m} O_{k}\right)$, and let

$$
\begin{gathered}
U^{m} \operatorname{Pic}(\mathfrak{X})=\operatorname{ker}\left(\operatorname{Pic}(\mathfrak{X}) \rightarrow \operatorname{Pic}\left(\mathfrak{X}_{m}\right)\right), \\
U^{m} \operatorname{Pic}(X)=\operatorname{Im}\left(U^{m} \operatorname{Pic}(\mathfrak{X}) \rightarrow \operatorname{Pic}(X)\right) .
\end{gathered}
$$


We define the Swan conductors of elements of $\operatorname{Br}(X)$ :

Definition 3.1. For $w \in \operatorname{Br}(X)$, we define the Swan conductor $\operatorname{sw}(w)$ of $w$ to be the non-negative integer

$$
\inf \left\{m \mid \operatorname{ker}\left(\langle\cdot, w\rangle_{X}\right) \supset U^{m+1} \operatorname{Pic}(X)\right\} .
$$

Now we begin the proof of Theorem 1.1. Let $T=H^{1}\left(Y_{e t}, i^{*} R j_{*} \mathbb{G}_{m}\right)$. We have a canonical isomorphism (cf. [5], 9.5)

$$
T \cong \operatorname{Coker}\left(\bigoplus_{\eta \in Y_{1}} K_{\eta}^{*} \rightarrow \bigoplus_{y \in Y_{0}}\left(\left(\bigoplus_{\eta_{y} \in Y_{1}^{y}} K_{\eta_{y}}^{*}\right) / R_{y}^{*}\right)\right) .
$$

For each integer $m>0$, let $U^{m} T$ be the image in $T$ of

$$
\bigoplus_{y \in Y_{0}} \bigoplus_{\eta_{y} \in Y_{1}^{y}}\left(1+\pi^{m} O_{K_{\eta_{y}}}\right) \text {. }
$$

Consider the natural homomorphism

$$
\psi: \operatorname{Pic}(X) \rightarrow T \text {. }
$$

Lemma 3.2. For each $m>0, \psi\left(U^{m} \operatorname{Pic}(X)\right) \subset U^{m} T$ and we have

$$
\operatorname{Pic}(X) / U^{m} \operatorname{Pic}(X) \stackrel{\cong}{\rightrightarrows} T / U^{m} T .
$$

Furthermore, the homomorphism

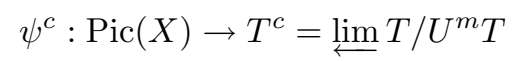

induced by $\psi$ is a homeomorphism, when we consider $T^{c}$ as a topological group by taking the image of $U^{m} T$ in $T^{c}$ for $m>0$ as a basis of neighborhoods at the origin. (Hence, $\left\{U^{m} \operatorname{Pic}(X)\right\}_{m>0}$ is a fundamental system of neighborhoods of $\operatorname{Pic}(X)$ at the origin.)

Proof. See [5] Lemma 9.8 and 9.10.

For $y \in Y_{0}$ and $\eta_{y} \in Y_{1}^{y}$, there exists a canonical isomorphism

$$
H^{3}\left(K_{\eta_{y}}, \mathbf{Q} / \mathbf{Z}(2)\right) \cong \mathbf{Q} / \mathbf{Z},
$$

which is given by two-dimensional local class field theory. Under this identification, we have for each $w \in \operatorname{Br}(X)$ the following diagram:

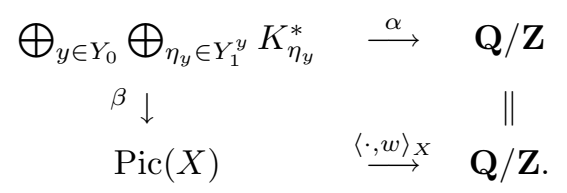

Here $\alpha$ is defined by

$$
\left(a_{y, \eta_{y}}\right) \mapsto \sum_{y \in Y_{0}} \sum_{\eta_{y} \in Y_{1}^{y}}\left\langle a_{y, \eta_{y}}, w_{K_{\eta_{y}}}\right\rangle_{K_{\eta_{y}}}
$$

where $w_{K_{\eta_{y}}}$ is the natural image of $w$ in $\operatorname{Br}\left(K_{\eta_{y}}\right)$, and $\beta$ is induced by $\psi^{c-1}$. This diagram is known to be anti-commutative (cf. [5] p. 411). We will briefly review the proof of this fact at the end of this section.

By Lemma 3.2, we have

$$
\beta\left(\bigoplus_{y \in Y_{0}} \bigoplus_{\eta_{y} \in Y_{1}^{y}}\left(1+\pi^{m} O_{K_{\eta_{y}}}\right)\right)=U^{m} \operatorname{Pic}(X) \quad(m>0) .
$$


This shows that

$$
\operatorname{sw}(w)=\sup \left\{\left[\operatorname{sw}_{K_{\eta_{y}}}\left(w_{K_{\eta_{y}}}\right) / e_{\eta}\right] \mid \eta \in Y_{1}, y \in\{\bar{\eta}\} \cap Y_{0}\right\},
$$

where $\eta_{y} \in Y_{1}^{y}$ is the unique element lying over $\eta$. By [3] Lemma 6.2, if $\eta \in Y_{1}$, for any $y \in\{\bar{\eta}\} \cap Y_{0}$ we have

$$
\mathrm{sw}_{K_{\eta_{y}}}\left(w_{K_{\eta_{y}}}\right)=\operatorname{sw}_{K_{\eta}}\left(w_{K_{\eta}}\right),
$$

and Theorem 1.1 follows.

From now on, we recall the proof of the anti-commutativity of (4). This is deduced from the following three facts and the definitions of (1) and (2). First, we have an explicit description of $\psi$ (cf. [5], Section 7). For each $y \in Y_{0}$, we have a composite map

$$
\psi_{y}: \bigoplus_{x \in X_{0}^{y}} \mathbf{Z} \longrightarrow \operatorname{Coker}\left[K_{y}^{*} \stackrel{\gamma}{\rightarrow}\left(\left(\bigoplus_{x \in X_{0}^{y}} \mathbf{Z}\right) \oplus\left(\bigoplus_{\eta_{y} \in Y_{1}^{y}} K_{\eta_{y}}^{*}\right)\right)\right] \stackrel{\cong}{\longleftarrow}\left(\bigoplus_{\eta_{y} \in Y_{1}^{y}} K_{\eta_{y}}^{*}\right) / R_{y}^{*} .
$$

Here $\gamma$ is defined to be the sum of the natural maps $K_{y}^{*} \rightarrow K_{\eta_{y}}^{*}\left(\eta_{y} \in Y_{1}^{y}\right)$ and the composite maps

$$
K_{y}^{*} \rightarrow K_{x}^{*} \stackrel{\operatorname{ord}_{K_{x}}}{\longrightarrow} \mathbf{Z} \quad\left(x \in X_{0}^{y}\right),
$$

where $\operatorname{ord}_{K_{x}}$ is the normalized valuation on $K_{x}$. Then the map $\psi$ is equal to the map induced by $\bigoplus_{y \in Y_{0}} \psi_{y}$ on $\operatorname{Pic}(X)$.

Secondly, the reciprocity law (cf. [5], 2.9) shows the following fact. Similar to (3), there exists for each closed point $x \in X$ a canonical isomorphism

$$
H^{3}\left(K_{x}, \mathbf{Q} / \mathbf{Z}(2)\right) \cong \mathbf{Q} / \mathbf{Z},
$$

which is again given by two-dimensional local class field theory. Under identifications (3) and (5), for $y \in Y_{0}, w \in \operatorname{Br}\left(K_{y}\right)$ and $a \in K_{y}^{*}$, we have an equation in $\mathbf{Q} / \mathbf{Z}$

$$
\sum_{x \in X_{0}^{y}}\left\langle a, w_{K_{x}}\right\rangle_{K_{x}}+\sum_{\eta_{y} \in Y_{1}^{y}}\left\langle a, w_{K_{\eta_{y}}}\right\rangle_{K_{\eta_{y}}}=0
$$

where $w_{K_{x}}$ is the natural image of $w$ in $\operatorname{Br}\left(K_{x}\right)$.

Finally, for a closed point $x \in X$ and $w \in \operatorname{Br}(X)$, we have a commutative diagram (cf. [5], 2.7)

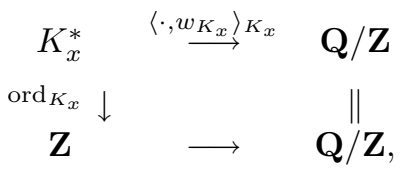

where the lower horizontal arrow is defined by the localization $w_{x} \in \operatorname{Br}(\kappa(x)) \cong$ $\mathbf{Q} / \mathbf{Z}$ of $w$ at $x$. Here we again used the identification (5).

\section{REFERENCES}

[1] Abhyankar, S., Resolution of singularities for arithmetical surfaces, In: Arithmetical Algebraic Geometry. New York. Harper and Row, 111-152 (1986) MR 34:171

[2] Hironaka, H, Desingularization of excellent surfaces, Lectures at Advanced Science Seminer in Algebraic Geometry. Bowdoin College, Summer 1967, noted by Bruce Bennett.

[3] Kato, K., Swan conductors for characters of degree one in the imperfect residue field case, Contemporary Math. 83,101-131 (1989) MR 90g:11164

[4] Lichtenbaum, S., Duality theorems for curves over p-adic fields, Invent. Math. 7, 120-136 (1969) MR 39:4158 
[5] Saito, S., Arithmetic on two dimensional local rings, Invent. Math. 85, 379-414 (1986) MR $\mathbf{8 7 j}: 11060$

[6] Yamazaki, T., Reduced norm map of division algebras over complete discrete valuation fields of certain type, to appear in Comp. Math.

Graduate School of Mathematical Sciences, University of Tokyo, Komaba 3-8-1, MEgro, TOKYO, 153 JAPAN

E-mail address: yama@ms406ss5.ms.u-tokyo.ac.jp 